\title{
NEW POINT CONFIGURATIONS AND ALGEBRAIC CURVES CONNECTED WITH THEM*
}

\author{
ARNOLD EMCH
}

1. Introduction. In the memorial volume $\dagger$ for Professor Hayashi, I studied an involutorial Cremona transformation in a projective $S_{r}$ which is obtained as follows: Let $C_{i}=(a x)_{i} \lambda_{i}{ }^{2}+(b x)_{i} \lambda_{i}+(c x)_{i}=0$, $(i=1,2, \cdots, r)$, be $r$ hypercones in $S_{r}$. Every value of $\lambda_{i}$ determines a hypertangent plane to the cone $C_{i}$. Thus the parameters $\lambda_{1}, \lambda_{2}, \cdots, \lambda_{r}$ for the hypercones $C_{1}, C_{2}, \cdots, C_{r}$, in the same order, determine $r$ hyperplanes which intersect in a point $(x)$ of $S_{r}$. From this point $(x)$ there pass, one for each of the $r$ hypercones, $r$ more tangent hyperplanes whose parameters $\lambda_{1}^{\prime}, \lambda_{2}^{\prime}, \cdots, \lambda_{r}^{\prime}$ are in the same order uniquely determined by the set $\lambda_{1}, \lambda_{2}, \cdots, \lambda_{r}$, and hence are rational functions

$$
\rho \lambda_{i}^{\prime}=\phi_{i}\left(\lambda_{1}, \lambda_{2}, \cdots, \lambda_{r}\right), \quad i=1,2, \cdots, r,
$$

of the parameters $\lambda$. Conversely, the set $\lambda_{1}^{\prime}, \lambda_{2}^{\prime}, \cdots, \lambda_{r}^{\prime}$ determines $\lambda_{i}$ uniquely: $\sigma \lambda_{i}=\phi_{i}\left(\lambda_{1}^{\prime}, \lambda_{2}^{\prime}, \cdots, \lambda_{r}^{\prime}\right)$. If therefore the $\lambda^{\prime}$ 's and $\lambda^{\prime \prime}$ 's are interpreted as coordinates of points of euclidean spaces $E_{r}(\lambda)$ and $E_{r}^{\prime}\left(\lambda^{\prime}\right)$, there exists an involutorial Cremona transformation between the two $r$-dimensional spaces. The order and fundamental elements of this involution were determined in the corresponding projective spaces $S_{r}$ and $S_{r}^{\prime}$ and applications given for $S_{2}$ and $S_{3}$. These belong to a remarkable class of involutions which have the property that when in $S_{r}$ and $S_{r}^{\prime}$

$$
P\left(\lambda_{1}, \lambda_{2}, \lambda_{3}, \cdots, \lambda_{r+1}\right), \quad P^{\prime}\left(\lambda_{1}^{\prime}, \lambda_{2}^{\prime}, \lambda_{3}^{\prime}, \cdots, \lambda_{r+1}^{\prime}\right)
$$

are corresponding points and any number of transpositions between coordinates in the same columns is performed, say

$$
\begin{aligned}
& Q\left(\lambda_{1}, \lambda_{2}^{\prime}, \lambda_{3}^{\prime}, \cdots, \lambda_{\iota}^{\prime}, \cdots, \lambda_{r}, \cdots, \lambda_{r+1}^{\prime}\right), \\
& Q^{\prime}\left(\lambda_{1}^{\prime}, \lambda_{2}, \lambda_{3}, \cdots, \lambda_{i}, \cdots, \lambda_{r}^{\prime}, \cdots, \lambda_{r+1}\right),
\end{aligned}
$$

then $Q, Q^{\prime}$ is always a couple of corresponding points of the involution.

To this class also belong the well known quadratic and cubic involutions in $S_{2}, \rho x_{i}^{\prime}=1 / x_{i},(i=1,2,3)$, and in $S_{3}, \rho x_{i}^{\prime}=1 / x_{i},(i=1,2,3,4)$,

* Presented to the Society, September 6, 1938.

$\dagger$ The Tôhoku Mathematical Journal, vol. 37 (1933), pp. 100-109. See also Commentarii Mathematici Helvetici, vol. 4 (1932), pp. 65-73. 
and in general in $S_{r}, \rho x_{i}^{\prime}=1 / x_{i},(i=1,2, \cdots, r+1)$. It is the purpose of this paper to show the importance of these in connection with the plane elliptic cubic in $S_{2}$ and a certain septimic of genus three in $S_{3}$.

2. The $\Delta_{8}$-configuration on the plane elliptic cubic. Let $A_{1}(1,0,0)$; $A_{2}(0,1,0) ; A_{3}(0,0,1) ; B(1,1,1) ; B_{1}(-1,1,1) ; B_{2}(1,-1,1)$; $B_{3}(1,1,-1)$ be the fundamental and invariant points of the quadratic involution in $S_{2}, T_{2} \equiv \rho x_{i}^{\prime}=1 / x_{i},(i=1,2,3)$, and perform the possible permutations between the coordinates of corresponding points as indicated above, so that we obtain the four couples of corresponding points

$$
\begin{array}{ll}
P_{1}\left(a_{1}, a_{2}, a_{3}\right), & P_{2}\left(a_{2} a_{3}, a_{2}, a_{3}\right), \\
P_{1}^{\prime}\left(a_{2} a_{3}, a_{3} a_{1}, a_{1} a_{2}\right), & P_{2}^{\prime}\left(a_{1}, a_{3} a_{1}, a_{1} a_{2}\right), \\
P_{3}\left(a_{1}, a_{3} a_{1}, a_{3}\right), & P_{4}\left(a_{1}, a_{2}, a_{1} a_{2}\right), \\
P_{3}^{\prime}\left(a_{2} a_{3}, a_{2}, a_{1} a_{2}\right), & P_{4}^{\prime}\left(a_{2} a_{3}, a_{3} a_{1}, a_{3}\right) .
\end{array}
$$

This is easily verified. Take for example $P_{2}$; its inverse is $\left(1 / a_{2} a_{3}\right.$, $\left.1 / a_{2}, 1 / a_{3}\right)$. Multiplying by $a_{1} a_{2} a_{3}$, we obtain as the same point $\left(a_{1}, a_{3} a_{1}, a_{1} a_{2}\right)$ which is $P_{2}^{\prime}$. The eight points of this $\Delta_{8}$ configuration can be grouped into couples whose joins pass through $A_{1}, A_{2}, A_{3}$ as indicated in the following table:

$$
\begin{aligned}
& A_{1}: P_{1} P_{2}, P_{1}^{\prime} P_{2}^{\prime}, P_{3} P_{4}^{\prime}, P_{3}^{\prime} P_{4} \\
& A_{2}: P_{1} P_{3}, P_{1}^{\prime} P_{3}^{\prime}, P_{2} P_{4}^{\prime}, P_{2}^{\prime} P_{4} \\
& A_{3}: P_{1} P_{4}, P_{1}^{\prime} P_{4}^{\prime}, P_{2} P_{3}^{\prime}, P_{2}^{\prime} P_{3} .
\end{aligned}
$$

It is moreover evident that the joins of corresponding points $P_{i} P_{i}^{\prime}$ pass through the point $O\left(a_{1}+a_{2} a_{3}, a_{2}+a_{3} a_{1}, a_{3}+a_{1} a_{2}\right)$. The result may be stated as the following theorem:

THEOREM 1. The eight points of a $\Delta_{8}$-configuration lie by twos on four lines through each of the points $A_{i}$. The joins of the four pairs of corresponding points pass through a fixed point $O$, uniquely determined by one pair of corresponding points in $a \Delta_{8}$.

Now $O$ is the isologue of an invariant elliptic cubic $C_{3}$ of the involution, uniquely determined by any pair $P_{i} P_{i}^{\prime}$ of $\Delta_{8}$. Of course, $C_{3}$ passes through $\Delta_{8}, B, B_{1}, B_{2}, B_{3}$, and also $O^{\prime}\left(a_{2} a_{3}, a_{3} a_{1}, a_{1} a_{2}\right)$. Draw any other secant $s$ through $O$ cutting $C_{3}$ in a pair $Q_{1} Q_{1}^{\prime}$ of corresponding points. These determine a new $\Delta_{8}$-configuration on the same $C_{3}$, so that there are $\infty^{1} \Delta_{8}$-configurations on $C_{3}$ attached to the triangle $A_{1} A_{2} A_{3}$. 
On any elliptic cubic $C_{3}$ there are $\infty^{1}$ Steinerian quadruples (points of tangency of four tangents from a point of $C_{3}$ to $C_{3}$ ) and for each a diagonal triangle $A_{1} A_{2} A_{3}$. Choose $A_{1} A_{2} A_{3}$ as the fundamental triangle* of a quadratic transformation and the Steinerian quadruple as the set of invariant points $B, B_{1}, B_{2}, B_{3}$. The elliptic cubic $C_{3}$ is invariant in this involution and the tangents at the $B$ 's cut $C_{3}$ in its isologue $O$. Hence we have the following theorem:

THEOREM 2. There are $\infty^{1} \Delta_{8}$-configurations on each of the diagonal triangles of the $\infty^{1}$ Steinerian quadruples of a given elliptic $C_{3}$, completely inscribed in this cubic.

3. The $\Delta_{16}$-configuration and a septimic of genus three in $S_{3}$ connected with it. (1) Consider the involutorial cubic transformation $T_{3}: \rho x_{i}^{\prime}=1 / x_{i},(i=1,2,3,4)$, in an $S_{3}$ with the fundamental points $A_{1}(1,0,0,0), A_{2}(0,1,0,0), A_{3}(0,0,1,0), A_{4}(0,0,0,1)$, and the invariant points $B_{i}( \pm 1, \pm 1, \pm 1, \pm 1),(i=1,2, \cdots, 8)$; and perform all possible permutations, or series of transpositions as explained in $\$ 1$. In this manner a configuration $\Delta_{16}$ of eight couples of corresponding points $P_{i} P_{i}^{\prime}$ is obtained as shown in the table

$$
\begin{array}{ll}
P_{1}\left(a_{1}, a_{2}, a_{3}, a_{4}\right) ; & P_{5}\left(a_{1}, a_{2}, a_{3}, a_{1} a_{2} a_{3}\right) ; \\
P_{1}^{\prime}\left(a_{2} a_{3} a_{4}, a_{1} a_{3} a_{4}, a_{1} a_{2} a_{4}, a_{1} a_{2} a_{3}\right) ; & P_{5}^{\prime}\left(a_{2} a_{3} a_{4}, a_{1} a_{3} a_{4}, a_{1} a_{2} a_{4}, a_{4}\right) ; \\
P_{2}\left(a_{2} a_{3} a_{4}, a_{2}, a_{3}, a_{4}\right) ; & P_{6}\left(a_{2} a_{3} a_{4}, a_{1} a_{3} a_{4}, a_{2}, a_{3}\right) ; \\
P_{2}^{\prime}\left(a_{1}, a_{1} a_{3} a_{4}, a_{1} a_{2} a_{4}, a_{1} a_{2} a_{3}\right) ; & P_{6}^{\prime}\left(a_{1}, a_{2}, a_{1} a_{2} a_{4}, a_{1} a_{2} a_{3}\right) ; \\
P_{3}\left(a_{1}, a_{1} a_{3} a_{4}, a_{3}, a_{4}\right) ; & P_{7}\left(a_{2} a_{3} a_{4}, a_{2}, a_{1} a_{2} a_{4}, a_{4}\right) ; \\
P_{3}^{\prime}\left(a_{2} a_{3} a_{4}, a_{2}, a_{1} a_{2} a_{4}, a_{1} a_{2} a_{3}\right) ; & P_{7}^{\prime}\left(a_{1}, a_{1} a_{3} a_{4}, a_{3}, a_{1} a_{2} a_{3}\right) ; \\
P_{4}\left(a_{1}, a_{2}, a_{1} a_{2} a_{4}, a_{4}\right) ; & P_{8}\left(a_{2} a_{3} a_{4}, a_{2}, a_{3}, a_{1} a_{2} a_{3}\right) ; \\
P_{4}^{\prime}\left(a_{2} a_{3} a_{4}, a_{1} a_{3} a_{4}, a_{3}, a_{1} a_{2} a_{3}\right) ; & P_{8}^{\prime}\left(a_{1}, a_{1} a_{3} a_{4}, a_{1} a_{2} a_{4}, a_{4}\right) .
\end{array}
$$

As in case of $S_{2}$ it may be verified at once that the points of each pair $P_{i} P_{i}^{\prime}$ correspond, and that the sixteen points lie by twos on eight lines through each $A_{i}$. If $Q$ is any point of $S_{3}$, then the line $A_{i} Q$ is transformed into the line $A_{i} Q^{\prime}$. The lines $P_{1} P_{2}, P_{3} P_{6}$ on $A_{1}$ and $P_{1} P_{3}, P_{2} P_{6}$ on $A_{2}$ form a quadrilateral in the plane $a_{4} x_{3}-a_{3} x_{4}=0 ; P_{1}^{\prime} P_{2}^{\prime}, P_{3}^{\prime} P_{6}^{\prime}$ on $A_{1}$, and $P_{1}^{\prime} P_{3}^{\prime}, P_{2}^{\prime} P_{6}^{\prime}$ on $A_{2}$ a quadrilateral in the conjugate plane $a_{3} x_{3}-a_{4} x_{4}=0$. The table of the thirty-two lines, eight through each $A_{i}$ follows :

* For $T_{2}, T_{3}$ and other involutions see the author's paper On surfaces and curves which are invariant under involutorial Cremona transformations, American Journal of Mathematics, vol. 48 (1926), pp. 21-44. 


$$
\begin{aligned}
& A_{1}: P_{1} P_{2}, P_{1}^{\prime} P_{2}^{\prime}, P_{3} P_{6}, P_{3}^{\prime} P_{6}^{\prime}, P_{4} P_{7}, P_{4}^{\prime} P_{7}^{\prime}, P_{5} P_{8}, P_{5}^{\prime} P_{8}^{\prime} ; \\
& A_{2}: P_{1} P_{3}, P_{1}^{\prime} P_{3}^{\prime}, P_{2} P_{6}, P_{2}^{\prime} P_{6}^{\prime}, P_{4} P_{8}^{\prime}, P_{4}^{\prime} P_{8}, P_{5} P_{7}^{\prime}, P_{5}^{\prime} P_{7} ; \\
& A_{3}: P_{1} P_{4}, P_{1}^{\prime} P_{4}^{\prime}, P_{2} P_{7}, P_{2}^{\prime} P_{7}^{\prime}, P_{3} P_{8}^{\prime}, P_{3}^{\prime} P_{8}, P_{5} P_{6}^{\prime}, P_{5}^{\prime} P_{6} ; \\
& A_{4}: P_{1} P_{5}, P_{1}^{\prime} P_{5}^{\prime}, P_{2} P_{8}, P_{2}^{\prime} P_{8}^{\prime}, P_{3} P_{7}^{\prime}, P_{3}^{\prime} P_{7}, P_{4} P_{6}^{\prime}, P_{4}^{\prime} P_{6} .
\end{aligned}
$$

It also appears at once that the eight joins of corresponding points $P_{i} P_{i}^{\prime}$ pass through a fixed point

$$
O\left(a_{1}+a_{2} a_{3} a_{4}, a_{2}+a_{1} a_{3} a_{4}, a_{3}+a_{1} a_{2} a_{4}, a_{4}+a_{1} a_{2} a_{3}\right) .
$$

To sum up we have the following theorem:

THEOREM 3. The sixteen points of $\Delta_{16}$ lie by twos on eight lines through each of the four $A_{i}$. The eight lines on each of any two of the four $A_{i}$ form four quadrilaterals on the chosen two $A_{i}$, which lie in two pairs of conjugate planes with the join of the two $A_{i}$ as a common axis. The joins of corresponding points of $\Delta_{16}$ pass through a fixed point $O$.

(2) It is known that the system of lines joining corresponding points of $T_{3}$ form a cubic line complex $\Gamma$ :

$$
p_{12} p_{13} p_{23}+p_{12} p_{14} p_{42}+p_{13} p_{14} p_{34}+p_{23} p_{42} p_{34}=0
$$

so that the lines of $\Gamma$ on a point $O\left(b_{1}, b_{2}, b_{3}, b_{4}\right), b_{1}=a_{1}+a_{2} a_{3} a_{4}, \cdots$, generate the cubic complex-cone

$$
\begin{aligned}
K= & \left(b_{1} x_{2}-b_{2} x_{1}\right)\left(b_{1} x_{3}-b_{3} x_{1}\right)\left(b_{2} x_{3}-b_{3} x_{2}\right) \\
& +\left(b_{1} x_{2}-b_{2} x_{1}\right)\left(b_{1} x_{4}-b_{4} x_{1}\right)\left(b_{4} x_{2}-b_{2} x_{4}\right) \\
& +\left(b_{1} x_{3}-b_{3} x_{1}\right)\left(b_{1} x_{4}-b_{4} x_{1}\right)\left(b_{3} x_{4}-b_{4} x_{3}\right) \\
& +\left(b_{2} x_{3}-b_{3} x_{2}\right)\left(b_{4} x_{2}-b_{2} x_{4}\right)\left(b_{3} x_{4}-b_{4} x_{3}\right)=0 .
\end{aligned}
$$

Among the generatrices of $K$ are the eight joins $P_{i} P_{i}^{\prime}$ of $\Delta_{16}$. The eight lines $O B_{i}$ lie on $K$. Any other generatrix $g$ of $K$ is on two corresponding points $Q$ and $Q^{\prime}$ of $T_{3}$. These determine another $\Delta_{16}$ uniquely, which also lies on $K$. Thus there are $\infty^{1} \Delta_{16}$ 's on $K$. Corresponding points $Q Q^{\prime}$ on $K$ form a certain space curve whose order is obtained as follows: The join of $Q(x), Q^{\prime}\left(x^{\prime}\right)$ passes through $O$ when

$$
\begin{array}{ll}
\lambda x_{1}+\mu x_{2} x_{3} x_{4}=b_{1}, & \lambda x_{2}+\mu x_{1} x_{3} x_{4}=b_{2}, \\
\lambda x_{3}+\mu x_{1} x_{2} x_{4}=b_{3}, & \lambda x_{4}+\mu x_{1} x_{2} x_{3}=b_{4} .
\end{array}
$$

Eliminating $\lambda, \mu, 1$ from any two distinct triples of these equations, say between the first three and the last three, the cubic cones $K_{4}$ and $K_{1}$ with vertices $A_{4}$ and $A_{1}$ and the common generatrix $A_{1} A_{4}$ are obtained, along which they have the common tangent plane $b_{2} x_{2}-b_{3} x_{3}$ 
$=0$. Hence they intersect in a residual septimic $C_{7}$, the locus of the point $Q, Q^{\prime}$. This follows immediately by inspection of the equations

$$
\begin{aligned}
& K_{4}=b_{1} x_{1}\left(x_{2}{ }^{2}-x_{3}{ }^{2}\right)+b_{2} x_{2}\left(x_{3}{ }^{2}-x_{1}{ }^{2}\right)+b_{3} x_{3}\left(x_{1}{ }^{2}-x_{2}{ }^{2}\right)=0, \\
& K_{1}=b_{2} x_{2}\left(x_{3}{ }^{2}-x_{4}^{2}\right)+b_{3} x_{3}\left(x_{4}{ }^{2}-x_{2}^{2}\right)+b_{4} x_{4}\left(x_{2}{ }^{2}-x_{3}{ }^{2}\right)=0 .
\end{aligned}
$$

This can be verified by other methods of proof which for the sake of brevity shall be omitted.

(3) To prove that the genus of $C_{7}$ is three, project $C_{7}$ upon $K_{1}$ from a generic point $P$. The projection proper is a residual $C_{14}$ of order $3 \times 7-7=14$. The cone $K_{4}$ cuts $C_{14}$ in $3 \times 14-6=36$ points proper, because $C_{7}$ touches both $K_{1}$ and $K_{4}$ along $A_{1} A_{4}$ in three points which accounts for six (improper) points of intersection. The polar conic of $P$ with respect to $K_{1}$ cuts $C_{7}$ outside of $A_{1}$ and $A_{4}$ in twelve points, so that altogether $36-12=24$ points of intersection are left which are projected into twelve double points of $C_{7}^{\prime}$, the projection of $C_{7}$ upon a generic plane. The genus $p$ of $C_{7}^{\prime}$ and hence of $C_{7}$ is therefore $p=6 \times 5 / 2-12=3$.

Now every couple $P_{i}^{\prime} P_{i}^{\prime}$ on $K$ or $C_{7}$ gives rise to a definite $\Delta_{16^{-}}$ configuration. Hence we have our next theorem:

TheOREM 4. On every cubic cone $K$ of the cubic complex associated with the involutorial cubic transformation $T_{3}$ there exists an invariant septimic $C_{7}$ of genus three with $\infty^{1} \Delta_{16}$-configurations.

It is interesting to note that the $C_{7}$ lies on two other cubic cones $K_{2}$ and $K_{3}$ with vertices at $A_{2}$ and $A_{3}$ by using the elimination process of $\lambda, \mu, 1$ in the remaining possible ways, so that it may also be characterized by the property that it lies on five cubic cones.

(4) The investigation may be extended to any other $S_{r}, r>3$, but this would amount merely to a simple generalization of the preceding theory.

UNIVERSITY OF ILLINOIS 